\title{
PREVALENCE AND RISK FACTORS OF SENSORY-NEURAL HEARING LOSS
}

\section{AMONG TELEPHONE OPERATORS.}

\author{
By \\ El-Bestar, S.F., El-Helaly, M.E. and Khashaba, E.O. \\ Community medicine department, Faculty of Medicine, Mansoura Univ., Egypt.
}

\begin{abstract}
:
Objectives: this study was carried out to evaluate the prevalence of sensory-neural hearing loss (SNHL) among telephone operators and its risk factors specially the effect of headset noise on the hearing ability of the telephone operators. Methods: a cross-sectional study was carried out on 58 telephone operators and 30 administration staff workers at Mansoura Telecommunications Company in Egypt. All participants were interviewed using a structured questionnaire including personal, medical and occupational history; and underwent an audiometric examination. Audiometric evaluation was done to evaluate hearing threshold levels (HTLs) for air and bone conduction for both right and left ears of all participants. Background noise was measured both at telephone operator and administration departments. Results: telephone operators had significantly higher prevalence of acoustic shock symptoms and decreased hearing sensitivity ( $46.6 \%$ and $37.9 \%$, respectively) compared to the controls $(3.3 \%$ and $13.3 \%$, respectively). Telephone operator had significantly higher HTLs compared to the controls, for air and bone conduction for both ears at lower and higher frequencies without the characteristic notch of noise induced hearing loss (NIHL). Among telephone operators, headset users had higher HTLs compared to headset non users, for air and bone conduction at the higher frequencies for both ears but more obviously in the left ear. There were 26 (44.8\%) cases of SNHL among the telephone operators versus no cases among the controls; all of them were bilateral in distribution. Among other studied factors, only headset use $(\mathrm{OR}=5.2,95 \% \mathrm{CI}=1.7$ 16.1) and age $(\mathrm{OR}=1.1,95 \% \mathrm{CI}=1.0-1.2)$ were significant risk factors for developing SNHL among telephone operators. Conclusion: telephone operators are exposed to
\end{abstract}


sudden repeated unexpected loud sounds due to headset use which could affect their hearing ability.

Key words: Hearing loss - Telephone operators - Headset - Prevalence- Risk Factors.

\section{Introduction}

Noise loading has considerable effect on the working conditions of telephone operators. Telephone operators are exposed to two types of noise, the back ground noise which can be attributed to densely occupied work rooms as well as to the socio- cultural behavior of the workers, namely speaking loudly; and the noise transmitted through the head set. However, the main source of noise exposure for telephone operators is likely to be through their headsets (Ivanovich et al., 1994)

In spite of the rapid growth in the communications industry in the last century, relatively little research has been published on occupational noise exposure of communication personnel. This lack of information has probably been due to the difficulties in the measurement set-up and in the assessment of the exposure itself. Moreover, telephone calls are typically verbal communications that, even if amplified, are considered a natural task and are not commonly associated to a hearing risk. Nevertheless, there is a large number of persons exposed to noise emitted from headsets and a high number of complaints are related to an over-exposure to this kind of noise (Planeau, 2005). Concerning Egypt, the Egyptian fixed line telecommunication services are among the fastest growing in the Middle East and North Africa (MENA), Arab, and African regions. By the end of the year 2000, Egypt's telecom revenued from the fixed line network alone amounted to over $\$ 2.5$ billion, representing $2 \%$ of Egypt's total GDP; this ranked Egypt second after Saudi Arabia in the Arab region (International Business Strategies, 2002).

Research by the UK's Health and Safety Executive showed that $30 \%$ of the interviewed telephone operators claimed symptoms of acoustic shock. Potentially this suggests that 300,000 UK operators may be acoustic shock victims (Smith, 2004). Moreover, Peretti et al. (2003) and Planeau (2005) carried out studies to evaluate noise levels emitted from headsets and concluded that the risk of hearing loss could exist for telephone operators if noise exposure levels exceeded $85 \mathrm{~dB}(\mathrm{~A})$. Therefore, this risk has to be carefully taken into account. 


\section{Subject and Methods}

\section{Study population}

Sixty telephone operators working at Mansoura Telecommunications Company were recruited and accepted to participate in our study. Two from the sixty telephone operators were excluded as they had a history of perforated ear drum. So, the study population consisted of 58 telephone operators whose work tasks were to respond to customer inquiries and complaints about non-working telephone lines and provide local and long distance calls to customers. Telephone operators' mean age was $46.3 \pm 8.1$ years and about two third $(63.8 \%)$ of them were females. They worked as telephone operators for a mean duration of 20.6 \pm 9.1 years and most of them were non-smoker (86.2\%). They were working a work shift was of 8 hours duration and they worked for 7 days per week. About half of them $(51.8 \%)$ used headset in making and answering calls; and the others did not use headsets.

Thirty administration staff workers at the Mansoura telecommunication company were recruited as a control group. Their mean age was $47.2 \pm 8.1$ years and about two third $(60.0 \%)$ of them were females. Their mean duration of employment was
$21.7 \pm 8.2$ years and most of them were nonsmoker $(90.0 \%)$.

\section{Exclusion criteria}

Subjects who had a history of perforated drum, impacted wax in their ears, history of ear surgery, firing guns and previous occupational exposure to high level of noise estimated to be greater than or equal to $85 \mathrm{~dB}(\mathrm{~A})$ were excluded

\section{Methods}

\section{Questionnaire:}

A structured questionnaire was applied to all the subjects. Its content included demographics (name, age, gender, residence, smoking habit and education level), occupational history (duration of employment, work tasks, headset use and occurrence of occupational accidents or illness), general health status (physical/mental symptoms, recent health complaints, and ergonomics problems), and ear symptoms (hearing deterioration, tinnitus, earache, ear discharge and history of acoustic shock)

\section{Clinical ear examination:}

This was done for all subjects to exclude any cause of conductive causes of hearing impairment such as wax or perforated drum. 


\section{Audiometric evaluation}

All participants underwent pure-tone audiometry tests using an audiometer (audiotur 710 portable pure tone audiometry). The process started with the right ear, ascending from $0.5,1,2,3,4$, 6 to $8 \mathrm{kHz}$, and then descending to 1 and $0.5 \mathrm{kHz}$. Each frequency began with 40 $\mathrm{dB}$ hearing loss. If a positive response was given by the subject, the signal was reduced by steps of $10 \mathrm{~dB}$ each, till the sound could not be heard. Then, a backward increase of intensity by $5 \mathrm{~dB}$ was given to identify the closest threshold for the specific frequency. If the subject could not hear at $40 \mathrm{~dB}$ hearing loss for a specific frequency at the very start, similar reverse processes were performed $(10 \mathrm{~dB}$ for each increase and 5 $\mathrm{dB}$ for each decrease). The left ear was then examined, when the right one was done. Frequency spectrum calibration in decibel hearing loss fulfilled the International Organization for Standardization (ISO) 8253-1 (ISO 8253-1, 1989) criteria for audiometric testing environment adapted to the American National Standards Institute (ANSI) S 3.6-1968 requirements (ANSI, 1970). Daily calibration was conducted before subjects were tested. Testing was performed in a soundproof audiometry room. Temporary threshold shift was eliminated, as the subjects were examined before starting work. Losses of hearing acuity exceeding $25 \mathrm{~dB}$ were considered abnormal (WHO, 2007). Only the cases of SNHL were included in the statistical analysis.

\section{Noise evaluation:}

Spot measurements of noise were determined with a sound level meter (452 sound level meter ANSI TYPE2, Scott instrument laboratories, USA. Serial number A6666435) adjusted on A weighting band. Electroacoustic calibration was performed before data collection every time. Noise was measured at the level of the ears, when the worker was sitting, at the site of each staff's desk). Three spot measurements of background noise were done both at the telephone operator department and the administration department, then the mean of measurements were calculated for each department. It was found that the mean background noise was $42.6 \pm 3.05 \mathrm{~dB}(\mathrm{~A})$ at the telephone operator department and $33.3 \pm 5.7 \mathrm{~dB}(\mathrm{~A})$ at the administration department. However, we could not measure the headset noise at this study as the Knowles Electronics manikin for Acoustuc Research (KEMAR) which 
was used for this purpose (Rice et al., 1987; Walles, 1988 and Loloyd, 1992) was not available for us.

\section{Statistical Methods}

Data entry and analysis design were accomplished using SPSS program version 16.0 under Windows. The results were statistically analyzed using chi-square test $(\chi 2)$ and Fisher's exact test for discrete data; and student t-test for parametric continuous variables. Mann-Whitney test was used for nonparametric continuous data. Analysis of data was performed using the 0.05 significance level and the 0.01 high significance level. Logistic regression was used to study risk factors of developing SNHL among telephone operators.

\section{RESULTS}

There was no statistically significant difference between the telephone operators and the controls as regard matching variables of age, gender, residence, education level, smoking and duration of employment $(\mathrm{P}>0.05)$. However, there was a statistically significant difference between the telephone operators and the controls regarding marital status $(\mathrm{P}<0.05)$. Telephone operators had statistically significant higher prevalence of acoustic shock symptoms and decreased hearing sensitivity (46.6\% and $37.9 \%$, respectively) compared to the controls $(3.3 \%$ and $13.3 \%$, respectively). However, there was no statistically significant difference between the telephone operators and the controls regarding other hearing symptoms such as tinnitus, loudness recruitment and ear pain (data were not tabulated).

The mean $\mathrm{dB}$ hearing loss for air conduction among telephone operators for both ears were significantly higher compared to the controls at both low and high frequencies (Table 1). The mean $\mathrm{dB}$ hearing loss for bone conduction among telephone operators was significantly higher compared to the controls both at low and high frequencies regarding both ears, however there was no statistically significant difference regarding the right ear at $8 \mathrm{KHz}$ (Table 2).

Concerning the effect of headset use on the hearing ability of the telephone operators, the mean $\mathrm{dB}$ hearing loss for air conduction for both ears were significantly higher $(\mathrm{P}<0.05)$ among telephone operator headset users compared to headset nonusers at the high frequencies, 4-8 KHz in the right ear and $2-8 \mathrm{KHz}$ in the left ear (Table 3). 
Also, the mean $\mathrm{dB}$ hearing loss for bone conduction were significantly higher in telephone operator headset users compared to headset non-users for the left ear at the high frequencies, 4-8 KHz. However, the bone conduction of the right ear of headset users showed significantly higher mean $\mathrm{dB}$ hearing loss in comparison to headset nonusers, only at 2,4 and $6 \mathrm{KHz}$ (Table 4).

There were $26(44.8 \%)$ cases of SNHL (all of them were bilateral in distribution) among the telephone operators versus no cases among the controls. Logistic regression analysis for developing SNHL among telephone operators, revealed that headset users were significantly at higher risk of SNHL compared to headset non-user $(\mathrm{OR}=5.2,95 \% \mathrm{CI}=1.7-16.1)$. Moreover, age had a significant OR for developing SNHL among telephone operators $(\mathrm{OR}=$ $1.1,95 \% \mathrm{CI}=1.0-1.2)($ Table 5$)$. 
Table (1): The mean $\mathrm{dB}$ hearing loss for air conduction among telephone operators $(n=58)$ and the controls $(n=30)$ for both ears.

\begin{tabular}{|c|c|c|c|c|c|}
\hline \multirow{2}{*}{$\begin{array}{l}\text { Frequency } \\
(\mathbf{K H z})\end{array}$} & \multirow{2}{*}{$\begin{array}{l}\text { Studied } \\
\text { groups }\end{array}$} & \multicolumn{2}{|c|}{$\begin{array}{c}\text { Right Ear } \\
\text { (air conduction) }\end{array}$} & \multicolumn{2}{|c|}{$\begin{array}{c}\text { Left Ear } \\
\text { (air conduction) }\end{array}$} \\
\hline & & $\begin{array}{c}\text { Mean } \pm \text { SD } \\
(\text { dB hearing loss) }\end{array}$ & p value & $\begin{array}{c}\text { Mean } \pm \text { SD } \\
(\text { dB hearing loss })\end{array}$ & $p$ value \\
\hline \multirow{2}{*}{0.5} & $\begin{array}{l}\text { Telephone } \\
\text { Operators }\end{array}$ & $47.8 \pm 8.9$ & \multirow{2}{*}{$\begin{aligned}(\mathrm{t} & =9.9) \\
& <0.01\end{aligned}$} & $45.8 \pm 8.3$ & \multirow{2}{*}{$\begin{aligned}(\mathrm{t} & =9.4) \\
& <0.01\end{aligned}$} \\
\hline & Controls & $25.6 \pm 7.5$ & & $26.0 \pm 7.8$ & \\
\hline \multirow{2}{*}{1} & $\begin{array}{l}\text { Telephone } \\
\text { Operators }\end{array}$ & $48.0 \pm 10.2$ & \multirow{2}{*}{$\begin{array}{l}(\mathrm{t}=8.7) \\
<0.01\end{array}$} & $43.4 \pm 10.1$ & \multirow{2}{*}{$\begin{array}{r}(\mathrm{t}=6.9) \\
<0.01\end{array}$} \\
\hline & Controls & $25.5 \pm 8.0$ & & $25.8 \pm 8.4$ & \\
\hline \multirow{2}{*}{2} & $\begin{array}{l}\text { Telephone } \\
\text { Operators }\end{array}$ & $41.8 \pm 9.5$ & \multirow{2}{*}{$\begin{array}{l}(\mathrm{t}=9.2) \\
<0.01\end{array}$} & $39.3 \pm 10.7$ & \multirow{2}{*}{$\begin{array}{c}(\mathrm{t}=7.4) \\
<0.01\end{array}$} \\
\hline & Controls & $21.2 \pm 8.6$ & & $21.4 \pm 7.1$ & \\
\hline \multirow{2}{*}{3} & $\begin{array}{l}\text { Telephone } \\
\text { Operators }\end{array}$ & $38.1 \pm 11.4$ & \multirow{2}{*}{$\begin{array}{l}(\mathrm{t}=7.6) \\
<0.01\end{array}$} & $37.5 \pm 12.6$ & \multirow{2}{*}{$\begin{array}{l}(\mathrm{t}=7.3) \\
<0.01\end{array}$} \\
\hline & Controls & $18.5 \pm 8.7$ & & $18.8 \pm 7.5$ & \\
\hline \multirow{2}{*}{4} & $\begin{array}{l}\text { Telephone } \\
\text { Operators }\end{array}$ & $35.2 \pm 13.9$ & \multirow[t]{2}{*}{$\begin{array}{c}\left(\mathrm{z}^{*}=5.2\right) \\
<0.01\end{array}$} & $34.0 \pm 14.3$ & \multirow[t]{2}{*}{$\begin{array}{c}(\mathrm{z}=4.8) \\
<0.01\end{array}$} \\
\hline & Controls & $20.3 \pm 8.8$ & & $19.6 \pm 8.9$ & \\
\hline \multirow{2}{*}{6} & $\begin{array}{l}\text { Telephone } \\
\text { Operators }\end{array}$ & $35.6 \pm 16.3$ & \multirow{2}{*}{$\begin{array}{l}(\mathrm{z}=3.6) \\
<0.01\end{array}$} & $34.6 \pm 17.9$ & \multirow{2}{*}{$\begin{array}{c}(\mathrm{z}=3.0) \\
<0.05\end{array}$} \\
\hline & Controls & $22.5 \pm 9.5$ & & $22.6 \pm 8.8$ & \\
\hline \multirow{2}{*}{8} & $\begin{array}{l}\text { Telephone } \\
\text { Operators }\end{array}$ & $36.8 \pm 21.3$ & \multirow{2}{*}{$\begin{array}{c}(z=3.6) \\
<0.01\end{array}$} & $35.0 \pm 17.4$ & \multirow{2}{*}{$\begin{array}{c}(\mathrm{z}=3.5) \\
<0.01\end{array}$} \\
\hline & Controls & $21.6 \pm 8.5$ & & $22.1 \pm 9.9$ & \\
\hline
\end{tabular}

$*_{\mathrm{z}}=$ Mann-Whitney test 
Table (2): The mean dB hearing loss for bone conduction among telephone operators $(n=58)$ and the controls $(n=30)$ for both ears.

\begin{tabular}{|c|c|c|c|c|c|}
\hline \multirow{2}{*}{$\begin{array}{l}\text { Frequency } \\
(\mathbf{K H z})\end{array}$} & \multirow{2}{*}{$\begin{array}{l}\text { Studied } \\
\text { groups }\end{array}$} & \multicolumn{2}{|c|}{$\begin{array}{c}\text { Right Ear } \\
\text { (bone conduction) }\end{array}$} & \multicolumn{2}{|c|}{$\begin{array}{c}\text { Left Ear } \\
\text { (bone conduction) }\end{array}$} \\
\hline & & $\begin{array}{c}\text { Mean } \pm \text { SD } \\
(\text { dB hearing loss })\end{array}$ & $p$ value & $\begin{array}{c}\text { Mean } \pm \text { SD } \\
(\mathrm{dB} \text { hearing loss })\end{array}$ & $p$ value \\
\hline \multirow[b]{2}{*}{0.5} & $\begin{array}{l}\text { Telephone } \\
\text { Operators }\end{array}$ & $45.2 \pm 11.2$ & \multirow{2}{*}{$\begin{array}{l}(\mathrm{t}=9.8) \\
<0.01\end{array}$} & $42.7 \pm 9.8$ & \multirow{2}{*}{$\begin{array}{c}(\mathrm{t}=0.00) \\
<0.01\end{array}$} \\
\hline & Controls & $24.0 \pm 6.7$ & & $21.8 \pm 6.6$ & \\
\hline \multirow[b]{2}{*}{1} & $\begin{array}{l}\text { Telephone } \\
\text { Operators }\end{array}$ & $44.0 \pm 10.5$ & \multirow{2}{*}{$\begin{array}{l}(\mathrm{t}=9.5) \\
<0.01\end{array}$} & $42.0 \pm 10.8$ & \multirow{2}{*}{$\begin{array}{c}(\mathrm{t}=0.00) \\
<0.01\end{array}$} \\
\hline & Controls & $22.8 \pm 9.2$ & & $21.3 \pm 7.3$ & \\
\hline \multirow[b]{2}{*}{2} & $\begin{array}{l}\text { Telephone } \\
\text { Operators }\end{array}$ & $46.0 \pm 10.2$ & \multirow{2}{*}{$\begin{array}{c}(\mathrm{t}=10.1) \\
<0.01\end{array}$} & $44.2 \pm 11.8$ & \multirow{2}{*}{$\begin{array}{c}(\mathrm{t}=0.00) \\
<0.01\end{array}$} \\
\hline & Controls & $24.5 \pm 7.8$ & & $23.3 \pm 9.5$ & \\
\hline \multirow[b]{2}{*}{3} & $\begin{array}{l}\text { Telephone } \\
\text { Operators }\end{array}$ & $44.1 \pm 10.5$ & \multirow{2}{*}{$\begin{array}{l}(\mathrm{t}=9.5) \\
<0.01\end{array}$} & $41.2 \pm 12.3$ & \multirow{2}{*}{$\begin{array}{c}(\mathrm{t}=0.00) \\
<0.01\end{array}$} \\
\hline & Controls & $23.1 \pm 7.6$ & & $22.8 \pm 7.3$ & \\
\hline \multirow[b]{2}{*}{4} & $\begin{array}{l}\text { Telephone } \\
\text { Operators }\end{array}$ & $35.7 \pm 8.8$ & \multirow{2}{*}{$\begin{array}{c}(\mathrm{t}=11.8) \\
<0.01\end{array}$} & $34.1 \pm 11.1$ & \multirow{2}{*}{$\begin{array}{c}(\mathrm{t}=0.00) \\
<0.01\end{array}$} \\
\hline & Controls & $17.6 \pm 6.9$ & & $18.3 \pm 6.6$ & \\
\hline \multirow[b]{2}{*}{6} & $\begin{array}{l}\text { Telephone } \\
\text { Operators }\end{array}$ & $28.1 \pm 12.7$ & \multirow{2}{*}{$\begin{array}{c}\left(\mathrm{z}^{*}=3.0\right) \\
<0.01\end{array}$} & $28.3 \pm 17.9$ & \multirow{2}{*}{$\begin{array}{c}(\mathrm{z}=2.9) \\
<0.01\end{array}$} \\
\hline & Controls & $21.0 \pm 9.6$ & & $18.5 \pm 6.8$ & \\
\hline \multirow[b]{2}{*}{8} & $\begin{array}{l}\text { Telephone } \\
\text { Operators }\end{array}$ & $29.9 \pm 27.1$ & \multirow{2}{*}{$\begin{array}{l}(\mathrm{z}=1.5) \\
>0.05\end{array}$} & $31.5 \pm 29.6$ & \multirow{2}{*}{$\begin{array}{c}(\mathrm{z}=2.5) \\
<0.01\end{array}$} \\
\hline & Controls & $21.5 \pm 13.7$ & & $16.0 \pm 7.2$ & \\
\hline
\end{tabular}

$*_{\mathrm{Z}}=$ Mann-Whitney test 
Table (3): The mean $\mathrm{dB}$ hearing loss for air conduction among headset users ( $\mathrm{n}=30)$ and headset non-user $(n=28)$ telephone operators for both ears.

\begin{tabular}{|c|c|c|c|c|c|}
\hline \multirow[b]{2}{*}{$\begin{array}{l}\text { Frequency } \\
\text { (KHz) }\end{array}$} & \multirow{2}{*}{$\begin{array}{l}\text { Telephone } \\
\text { operators }\end{array}$} & \multicolumn{2}{|c|}{$\begin{array}{c}\text { Right Ear } \\
\text { (air conduction) }\end{array}$} & \multicolumn{2}{|c|}{$\begin{array}{c}\text { Left Ear } \\
\text { (air conduction) }\end{array}$} \\
\hline & & $\begin{array}{c}\text { Mean } \pm \text { SD } \\
\text { (dB hearing } \\
\text { loss })\end{array}$ & $p$ value & $\begin{array}{c}\text { Mean } \pm \text { SD } \\
(\mathrm{dB} \text { hearing } \\
\text { loss })\end{array}$ & $p$ value \\
\hline \multirow{2}{*}{0.5} & $\begin{array}{c}\text { Headset } \\
\text { Users }\end{array}$ & $48.6 \pm 7.5$ & \multirow{2}{*}{$\begin{array}{l}(\mathrm{t}=0.7) \\
>0.05\end{array}$} & $47.0 \pm 9.05$ & \multirow{2}{*}{$\begin{array}{l}(t=0.9) \\
>0.05\end{array}$} \\
\hline & $\begin{array}{c}\text { Headset } \\
\text { Non-users }\end{array}$ & $47.03 \pm 10.6$ & & $44.8 \pm 7.5$ & \\
\hline \multirow{2}{*}{1} & $\begin{array}{l}\text { Headset } \\
\text { Users }\end{array}$ & $48.5 \pm 8.6$ & \multirow{2}{*}{$\begin{array}{l}(\mathrm{t}=0.3) \\
>0.05\end{array}$} & $45.1 \pm 10.6$ & \multirow{2}{*}{$\begin{array}{l}(t=1.3) \\
>0.05\end{array}$} \\
\hline & $\begin{array}{c}\text { Headset } \\
\text { Non-users }\end{array}$ & $47.5 \pm 12.03$ & & $41.8 \pm 9.3$ & \\
\hline \multirow{2}{*}{2} & $\begin{array}{c}\text { Headset } \\
\text { Users }\end{array}$ & $42.3 \pm 8.7$ & \multirow{2}{*}{$\begin{array}{l}(\mathrm{t}=0.3) \\
>0.05\end{array}$} & $41.8 \pm 12.2$ & \multirow{2}{*}{$\begin{array}{l}(\mathrm{t}=1.9) \\
<0.05\end{array}$} \\
\hline & $\begin{array}{l}\text { Headset } \\
\text { Non-users }\end{array}$ & $41.4 \pm 10.5$ & & $36.2 \pm 8.2$ & \\
\hline \multirow{2}{*}{3} & $\begin{array}{c}\text { Headset } \\
\text { Users }\end{array}$ & $38.5 \pm 11.2$ & \multirow{2}{*}{$\begin{array}{l}(\mathrm{t}=0.4) \\
>0.05\end{array}$} & $40.8 \pm 14.1$ & \multirow{2}{*}{$\begin{array}{l}(\mathrm{t}=2.3) \\
<0.01\end{array}$} \\
\hline & $\begin{array}{c}\text { Headset } \\
\text { Non-users }\end{array}$ & $37.2 \pm 11.7$ & & $33.5 \pm 9.5$ & \\
\hline \multirow{2}{*}{4} & $\begin{array}{c}\text { Headset } \\
\text { Users }\end{array}$ & $37.3 \pm 12.6$ & \multirow{2}{*}{$\begin{aligned}\left(\mathrm{z}^{*}\right. & =2.8) \\
& <0.05\end{aligned}$} & $39.1 \pm 14.3$ & \multirow[b]{2}{*}{$\begin{array}{c}(\mathrm{z}=4.1) \\
<0.01\end{array}$} \\
\hline & $\begin{array}{c}\text { Headset } \\
\text { Non-users }\end{array}$ & $32.2 \pm 14.6$ & & $27.9 \pm 12.1$ & \\
\hline \multirow{2}{*}{6} & $\begin{array}{c}\text { Headset } \\
\text { Users }\end{array}$ & $37.8 \pm 16.3$ & \multirow{2}{*}{$\begin{array}{r}(\mathrm{z}=2.8) \\
\quad<0.01\end{array}$} & $39.1 \pm 17.7$ & \multirow[b]{2}{*}{$\begin{array}{c}(\mathrm{z}=3.5) \\
<0.01\end{array}$} \\
\hline & $\begin{array}{c}\text { Headset } \\
\text { Non-users }\end{array}$ & $32.4 \pm 15.5$ & & $28.1 \pm 14.7$ & \\
\hline \multirow{2}{*}{8} & $\begin{array}{c}\text { Headset } \\
\text { Users }\end{array}$ & $40.6 \pm 22.8$ & \multirow{2}{*}{$\begin{array}{r}(\mathrm{z}=3.3) \\
\quad<0.01\end{array}$} & $40.1 \pm 18.1$ & \multirow{2}{*}{$\begin{array}{c}(\mathrm{z}=3.9) \\
<0.01\end{array}$} \\
\hline & $\begin{array}{c}\text { Headset } \\
\text { Non-users }\end{array}$ & $31.6 \pm 18.29$ & & $28.5 \pm 14.4$ & \\
\hline
\end{tabular}


Table (4): The mean $\mathrm{dB}$ hearing loss for bone conduction among headset users ( $n=30$ ) and headset non-user $(n=28)$ telephone operators for both ears.

\begin{tabular}{|c|c|c|c|c|c|}
\hline \multirow{2}{*}{$\begin{array}{l}\text { Frequency } \\
(\mathrm{KHz})\end{array}$} & \multirow{2}{*}{$\begin{array}{l}\text { Studied } \\
\text { groups }\end{array}$} & \multicolumn{2}{|c|}{$\begin{array}{c}\text { Right Ear } \\
\text { (bone conduction) }\end{array}$} & \multicolumn{2}{|c|}{$\begin{array}{c}\text { Left Ear } \\
\text { (bone conduction) }\end{array}$} \\
\hline & & $\begin{array}{c}\text { Mean } \pm \mathrm{SD} \\
(\mathrm{dB} \text { hearing } \\
\text { loss })\end{array}$ & $\mathrm{p}$ value & $\begin{array}{c}\text { Mean } \pm \text { SD } \\
(\mathrm{dB} \text { hearing } \\
\text { loss })\end{array}$ & $\mathrm{p}$ value \\
\hline \multirow{2}{*}{0.5} & $\begin{array}{c}\text { Headset } \\
\text { Users }\end{array}$ & $48.8 \pm 12.1$ & \multirow{2}{*}{$\begin{array}{l}(\mathrm{t}=2.4) \\
<0.01\end{array}$} & $47.7 \pm 8.5$ & \multirow{2}{*}{$\begin{array}{l}(\mathrm{t}=3.8) \\
<0.01\end{array}$} \\
\hline & $\begin{array}{c}\text { Headset } \\
\text { Non-users } \\
\end{array}$ & $41.5 \pm 9.3$ & & $38.2 \pm 8.9$ & \\
\hline \multirow{2}{*}{1} & $\begin{array}{l}\text { Headset } \\
\text { Users }\end{array}$ & $46.0 \pm 9.3$ & \multirow{2}{*}{$\begin{array}{l}(\mathrm{t}=1.8) \\
>0.05\end{array}$} & $44.8 \pm 10.2$ & \multirow{2}{*}{$\begin{array}{l}(\mathrm{t}=1.9) \\
>0.05\end{array}$} \\
\hline & $\begin{array}{c}\text { Headset } \\
\text { Non-users }\end{array}$ & $41.1 \pm 11.07$ & & $39.2 \pm 11.1$ & \\
\hline \multirow{2}{*}{2} & $\begin{array}{c}\text { Headset } \\
\text { Users }\end{array}$ & $47.3 \pm 11.5$ & \multirow{2}{*}{$\begin{array}{l}(\mathrm{t}=1.1) \\
<0.05\end{array}$} & $46.07 \pm 11.8$ & \multirow{2}{*}{$\begin{array}{l}(\mathrm{t}=1.2) \\
>0.05\end{array}$} \\
\hline & $\begin{array}{c}\text { Headset } \\
\text { Non-users } \\
\end{array}$ & $44.2 \pm 8.2$ & & $42.1 \pm 11.6$ & \\
\hline \multirow{2}{*}{3} & $\begin{array}{c}\text { Headset } \\
\text { Users }\end{array}$ & $45.6 \pm 11.1$ & \multirow{2}{*}{$\begin{array}{l}(\mathrm{t}=1.2) \\
>0.05\end{array}$} & $43.5 \pm 12.8$ & \multirow{2}{*}{$\begin{array}{l}(\mathrm{t}=1.5) \\
>0.05\end{array}$} \\
\hline & $\begin{array}{c}\text { Headset } \\
\text { Non-users } \\
\end{array}$ & $42.3 \pm 9.7$ & & $38.4 \pm 11.5$ & \\
\hline \multirow{2}{*}{4} & $\begin{array}{c}\text { Headset } \\
\text { Users }\end{array}$ & $38.0 \pm 9.3$ & \multirow{2}{*}{$\begin{array}{l}(\mathrm{t}=2.1) \\
<0.05\end{array}$} & $37.1 \pm 11.4$ & \multirow{2}{*}{$\begin{array}{l}(\mathrm{t}=2.4) \\
<0.05\end{array}$} \\
\hline & $\begin{array}{c}\text { Headset } \\
\text { Non-users } \\
\end{array}$ & $33.07 \pm 7.6$ & & $30.3 \pm 9.2$ & \\
\hline \multirow{2}{*}{6} & $\begin{array}{c}\text { Headset } \\
\text { Users }\end{array}$ & $28.1 \pm 12.06$ & \multirow{2}{*}{$\begin{array}{c}\left(\mathrm{z}^{*}=2.1\right) \\
<0.05\end{array}$} & $33.9 \pm 21.7$ & \multirow{2}{*}{$\begin{array}{c}(\mathrm{z}=4.1) \\
<0.01\end{array}$} \\
\hline & $\begin{array}{c}\text { Headset } \\
\text { Non-users } \\
\end{array}$ & $27.8 \pm 13.7$ & & $22.3 \pm 10.6$ & \\
\hline \multirow{2}{*}{8} & $\begin{array}{c}\text { Headset } \\
\text { Users }\end{array}$ & $33.9 \pm 29.3$ & \multirow{2}{*}{$\begin{array}{l}(\mathrm{z}=1.4) \\
>0.05\end{array}$} & $34.1 \pm 29.8$ & \multirow{2}{*}{$\begin{array}{l}(\mathrm{z}=2.8) \\
<0.01\end{array}$} \\
\hline & $\begin{array}{l}\text { Headset } \\
\text { Non-users }\end{array}$ & $26.5 \pm 25.5$ & & $29.0 \pm 30.3$ & \\
\hline
\end{tabular}

$*_{\mathrm{z}}=$ Mann-Whitney test 
Table (5): Logistic regression analysis and odds ratio (OR) for developing SNHL among telephone operators, according to headset use, age, sex, smoking, BMI and duration of employment.

\begin{tabular}{|c|c|c|c|c|c|}
\hline \multirow{3}{*}{ Variable } & \multicolumn{2}{|c|}{ Telephone Operators } & \multirow{3}{*}{ OR } & \multirow{3}{*}{$\begin{array}{c}95 \% \\
\text { Confidence } \\
\text { Interval }\end{array}$} & \multirow{3}{*}{ p Value } \\
\hline & $\begin{array}{l}\text { SNHL* }^{*} \\
(26)\end{array}$ & $\begin{array}{c}\text { Normal } \\
(32)\end{array}$ & & & \\
\hline & $\mathbf{N}(\%) /$ Mean \pm SD & $\mathrm{N}(\%) /$ Mean $\pm \mathrm{SD}$ & & & \\
\hline $\begin{array}{l}\text { Headset use } \\
\text { - no } \\
\text { - yes }\end{array}$ & $\begin{array}{c}7(26.9 \%) \\
19(73.1 \%)\end{array}$ & $\begin{array}{l}21(65.6 \%) \\
11(34.4 \%)\end{array}$ & 5.2 & $1.7-16.1$ & $<0.01$ \\
\hline Age & $49.3 \pm 6.8$ & $43.9 \pm 8.3$ & 1.1 & $1.0-1.2$ & $<0.05$ \\
\hline $\begin{array}{l}\text { Sex } \\
\text {-male } \\
\text {-female }\end{array}$ & $\begin{array}{l}11(43.3 \%) \\
15(57.7 \%)\end{array}$ & $\begin{array}{l}10(31.2 \%) \\
22(68.8 \%)\end{array}$ & 1.6 & $0.5-4.7$ & $>0.05$ \\
\hline $\begin{array}{l}\text { Smoking } \\
\text {-non-smoker } \\
\text {-smoker }\end{array}$ & $\begin{array}{c}23(88.5 \%) \\
3(11.5 \%)\end{array}$ & $\begin{array}{c}27(84.4 \%) \\
5(15.6 \%)\end{array}$ & 1.4 & $0.3-6.6$ & $>0.05$ \\
\hline BMI & $31.4 \pm 6.2$ & $30.6 \pm 4.8$ & 1.02 & $0.9-1.1$ & $>0.05$ \\
\hline $\begin{array}{l}\text { Duration of } \\
\text { employment }\end{array}$ & $22.8 \pm 8.7$ & $18.8 \pm 9.1$ & 1.1 & $0.9-1.1$ & $>0.05$ \\
\hline
\end{tabular}

*There were $26(44.8 \%)$ cases of SNHL among the telephone operators versus no cases among the controls; all of them were bilateral in distribution. 


\section{Discussion}

In the present study, the mean background noise was $42.6 \pm 3.05 \mathrm{~dB}$ (A) at the telephone operator department. This level was comparable to the acceptable levels of 45 or $50 \mathrm{dBA}$ recommended for similar work activities such as office work and VDT operator (Roussel, 1983). However other studies reported higher intensities of the background noise levels at telephone operators working rooms but did not exceed $85 \mathrm{~dB}$ (A) (Ivanovich, 1994; Patel and Broughton, 2002). The background noise could be attributed mainly to general conversation of telephone operators with callers and colleagues and to a lesser extent to other sources of noise such as telephone ringing. However, headset noise was not measured at this study as the Knowles Electronics manikin for Acoustic Reseach (KEMAR) used for this purpose (Rice et al. 1987; Walles, 1988 and Loloyd, 1992) was not available for us at the time of the study.

Regarding hearing symptoms, telephone operators had higher prevalence of acoustic shock symptoms and had greater hearing loss $(46.6 \%$ and $37.9 \%$, respectively) compared to the controls (3.3\% and $13.3 \%$, respectively). The acoustic shock symptoms among telephone operators were wok-related; however, the acoustic shock symptoms among controls were not work-related. This is in accordance with other studies which reported frequent acoustic shock symptoms among telephone operators (Smith, 2004; Planeau, 2005). The International Telecommunication Union (1998) stated that telephone operators were the workers most at risk for acoustic shock. This could be explained by that most of the telephone operators at the present study who were using headsets (51.8\%) did not use the headset volume control regularly and were exposed to sudden unexpected loud sounds randomly transmitted via the telephone lines (Westcott, 2006).

Moreover, Patuzzi et al. (2000) and Milhinch and Doyle (2000) investigated symptoms that allegedly resulted from exposure to acoustic shock events. These include tinnitus, pain, hypersensitivity to sound, vertigo, numbness/tenderness/ soreness around the ear and neck; headache and fatigue. They explained that the levels of noise transmitted through the headsets were not capable of damaging the ear directly. It is more likely that the trauma is caused by excessive middle ear contractions which were triggered by stress and anxiety. Additional stress can make the situation worse by lowering the threshold for these contractions. 
The audiometric evaluation of air and bone conduction of the study population revealed that telephone operators had significantly higher mean $\mathrm{dB}$ hearing loss compared to the controls for both ears, at low frequencies and most of the high frequencies. Moreover, according to WHO diagnostic criteria of SNHL (WHO, 2007), there were 26 (44.8\%) cases of SNHL among the telephone operators versus no cases among the controls. All cases of SNHL among telephone operators were bilateral in distribution without the characteristic notch of the noise induced hearing loss.

Concerning the source of exposure to noise among telephone operators, the measured background noise did not exceed the $85 \mathrm{~dB}$ threshold level to be a cause of the diagnosed SNHL. This study did not measure the headset noise; however, the telephone operators using headsets had significantly higher mean $\mathrm{dB}$ hearing loss in comparison to those not using headsets, at the high frequencies for both ears, more obviously at the left ear. This could be explained by the fact that most headset users were using the headset by their left ear. Moreover, among other studied factors, logistic regression analysis revealed that only headset use $(\mathrm{OR}=5.2,95 \% \mathrm{CI}=1.7$ 16.1) and age $(\mathrm{OR}=1.1,95 \% \mathrm{CI}=1.0-1.2)$ were significant risk factors for developing SNHL among telephone operators.

These results suggest that the main source of noise exposure for telephone operators at the present study was headset noise. The sources of noise transmitted through the headset could be callers with loud voice or calls made from noisy environments such as shops and homes with crying children (Ivanovich, 1994; Clark and Bohne, 1999 and Patel and Broughton, 2002).

Our results support other studies (Beastall, 1992; Williams and Presbury, 2003) which reported that headset use may produce SNHL either temporarily or permanently due to damage in the outer hair cells of the cochlea, but without the characteristic notch. Moreover, other authors (Peretti, 2003; Planeau, 2005) evaluated noise levels emitted from headsets and concluded that the risk of hearing loss could exist for telephone operators if noise exposure levels exceeded $85 \mathrm{~dB}$ (A). Furthermore, it has long been suspected that individuals who work professionally in industries where they must wear headphones in order to listen to either incoming or outgoing communication signals are at risk of noise injury and subsequent hearing loss (Williams and Presbury, 2003). However, these results contradict the studies reported 
by other authors (Ivanovich, 1994; Patel and Broughton, 2002) who reported that the risk of hearing damage from headsets was minimal.

Concerning other factors which could be related to developing SNHL among telephone operators at the present study, age was a significant risk factor; however, OR was near to one $(\mathrm{OR}=1.1,95 \% \mathrm{CI}=$ 1.0-1.2). Gates et al. (2000) reported that an interesting interaction between NIHL and age-related hearing loss has been found. Smoking was not a risk factor for developing SNHL at the present study which could be explained by the fact that $63.8 \%$ of telephone operators were females who did not smoke. Other studied factors including gender, BMI and duration of employment were not associated with SNHL.

\section{Conclusion}

The telephone operators are exposed to sudden repeated loud sounds and acoustic shocks due to headset use which could affect their hearing ability. This calls for more technical protection, training on the proper use of headset, and periodic audiometric evaluation of the telephone operators to prevent the occurrence of SNHL among them.

\section{References}

1. ANSI (1970). American National Standard Specifications for Audiometers. ANSIS3 .6-1969. American National Standards Institute Inc, New York.

2. Beastall, R.H. (1992). Acute acoustic trauma in a telephone operator. Occup Med 42 :215-216.

3. Clark, W. and Bohne, B. (1999). Effects of Noise on Hearing. J Am Med Asso 281:16581659.

4. Gates, G.A., Schmid, P., Kujawa, S.G. (2000). Longitudinal threshold changes in older men with audiometric notches. Hear Res.; 141(12):220-8.

5. International Business Strategies (2002). Telecommunications Equipment and Services in Egypt. Retrieved Jan, 8, 2007, from www. International business strategies .com.

6. International Telecommunication Union (ITU) (1998). ITU Recommendation.Vocabulary of terms on telephone transmission quality and telephone sets, P.10 (12/98). Geneva.

7. ISO 8253-1 (1989). Acoustics-Audiometric Test Methods - Part 1: Basic Pure Tone Air and Bone Conduction Audiometry. International Organization for Standardization, Geneva.

8. Ivanovich, E., Kolarova, D., Enev, S. (1994). Noise evaluation and estimation of some specific and non-specific health indicators in telephone operators. Rev. Environ. Health 10(1):39-46.

9. Loloyd, J.A. (1992). An assessment of the methods for measuring noise exposure from headsets. HSL Internal Report IR/L/NV/92/16. Buxton: Health \& Safety Laboratory.

10. Macrae, J.H. (1995). Hearing conservation standards for occupational noise exposure of workers from headphones or insert earphones. Aust. J. Audiol 17 (2): 107-114.

11. Milhinch, J. and Doyle, J. (2000). Acute aural trauma in headset and handset users. Paper presented at 14th National Conference of the 
Audiological Society of Australia, Adelaide, Australia.

12. Patel J.A. and Broughton, K. (2002). Assessment of the Noise Exposure of Call Centre Operators. Ann. occup. Hyg. 46(8):653 661.

13. Patuzzi, R., Milhinch, J. and Doyle, J. (2000). Acute aural trauma in telephone headset and handset users. Paper presented at the Neuro - Otological Society of Australia National Conference, Melbourne, Australia.

14. Peretti, A., Pedrielli, M., Baiamonte, F. (2003). Headphone Noise: Occupational Noise Exposure Assessment for Communication Personnel. Euronoise Naples,paperID:365-IP Retrieved May,15,2006, from http://www.pcfarina.eng. unipr.it/Public/Papers/181Euronoise2003.PDF

15. Planeau, V. (2005). Noise hazards associated with the call centre industry. euronoise. Retrieved Jan, 10, 2006, from http://www.osha. europa .eu/ european noisesummit /speeches / ew05_planeau.pdf

16. Rice, C.G., Breslin, M. and Roper, R.G. (1987). Sound levels from personal cassette players . Br. J. Audiol; 21: 273-8.
17. Roussel, C., Argence-tramunt, C. and Vial, F. (1983). Background noise in the data processing and office environment. Arch. d Malad Prof. 44(8):559-63.

18. Smith, S. (2004). Call Center Noise Hazards Place Operators at Risk. Retrieved Feb14,2007,from http:// www.occupationalhazards .com/Issue /Article/ 37279/ Call_Center_ Noise_Hazards_Place_Operators_at_Risk.aspx

19. Walles, E.J. (1988). Sound pressure levels from telephonists' headsets at Greater Manchester Fire Headquarters. HSL Internal Report IR/L/ NV/88/16. Buxton: Health \& Safety Laboratory.

20. Westcott, M. (2006). Acoustic shock injury (ASI). Acta. Otolaryngol. Suppl. 556:54-8.

21. Williams, W. and Presbury, J. (2003). Observations of noise exposure through the use of headphones by radio announcers. Noise Health 5:69-73

22. World Health Organization (WHO) (2001). Occupational and community noise. World Health Organization (Fact Sheet No. 258), Geneva. 